\title{
Allergy Services at a Tertiary Referral Centre: A One Year Retrospective Review of All Referrals "A One Year Review of Allergy Referrals"
}

Andrew Swindlehurst ${ }^{1 *}$, Nicola Swindlehurst ${ }^{1}$, Sunny Kaul ${ }^{2}$ and Christopher Corrigan ${ }^{3}$

${ }^{1}$ Maidstone and Tunbridge Wells NHS Trust, Maidstone Hospital Postgrad Centre, UK

${ }^{2}$ Royal Brompton and Harefield NHS Foundation Trust, The Harefield Hospital, UK

${ }^{3}$ Guy's and St Thomas' NHS Foundation Trust, Guys Hospital, London

\section{Introduction}

Allergic diseases affect approximately one third of the UK population and place an economic burden on the NHS amounting to over $£ 1$ billion per year [1,2]. There is an increasing prevalence within the UK of allergic rhinitis, nut allergy, anaphylaxis, occupational allergy and drug allergy with the UK ranking highly when compared with other developed countries [3].

Despite this rising prevalence, allergy services in the UK have been described as "totally inadequate" by the Royal College of Physicians [3], a conclusion based partly on information in the Healthcare Commission report "Provision of Allergy Services" in 2003/04 [4]. The report indicated that NHS allergy services had not kept pace with the growth of allergic disease, and also pointed to the patchiness of the service, with only 6 major centres staffed by consultant allergists offering a full-time service with expertise in all types of allergic problems and a further 9 centres staffed by allergists offering a part-time service. The remaining 86 allergy clinics in the UK are run part-time by consultants in other medical specialties.

The increasing prevalence of allergic disease, inadequacy of specialist services and the recent Department of Health initiative to manage more patients in the community [5] all increase the imperative that primary care physicians should be empowered by knowledge and experience to manage allergic disease where practicable, but if not to make referrals which are minimal in number but optimal in appropriateness for specialist allergy care. In the meantime, given the contribution of allergic conditions to the symptomatology and differential diagnosis of a wide range of disorders seen by experts in other specialities such as respiratory medicine, ENT, gastroenterology and dermatology [6], the modern day clinical allergist must have an astute awareness of such conditions and training must be tailored to suit.

There are no recent data concerning referrals to specialist UK allergy centres. The present study examines all referrals to one centre over an entire year and explores the diagnostic concordance between referral sources and allergists (a crude but potential marker of referral appropriateness) and the range of diagnoses that result (referral breadth). If recapitulated at other centres the data will inform strategies for increasing appropriateness of referrals and identify training needs for physicians who make referrals to allergy centres as well as the allergy physicians who receive them.

\section{Materials and Methods}

\section{Design}

Retrospective, all inclusive annual review.

\section{Setting}

Adult allergy service, Guy's and St Thomas' NHS Foundation Trust, London.

\section{Materials}

Anonymised data on all 1,702 new referrals made in 2006.

\section{Methods}

Information was obtained from the GSTT appointment booking system. Two authorised researchers systematically extracted information from each patient's clinic letters and investigations obtained from the Electronic Patient Records (EPR) health information system and converted this into categorical data recorded anonymously on an excel spreadsheet. Patient identifiable data were not stored or transferred anywhere else. Strict criteria were used to define each category in advance.

Data

Data extracted and recorded included:

1. Age and sex (from the clinic letter).

2. Source of referral (from the clinic letter).

3. Presenting complaints and referral diagnosis (from the clinic letter).

4. Allergist's working diagnosis based on patient assessment at first outpatient appointment and clinic letter. In all cases (including where there was more than one diagnosis) the diagnosis for the main presenting symptom was used (for example if a patient had symptoms of seasonal allergic rhinitis but also mentioned symptoms of an allergy to food then seasonal allergic rhinitis was listed as the working diagnosis). If a differential diagnosis was listed, the diagnosis that the allergist deemed most likely to be causing the patient's symptoms was used. This was usually apparent from the clinic letter.

5. All investigations requested by the allergist including blood tests, skin prick tests, allergen challenges and imaging and the results (EPR).

6. Final diagnosis (from clinic letter written by the allergist to the referring doctor). In the event that there were multiple final

*Corresponding author: Andrew Swindlehurst, Maidstone and Tunbridge Wells NHS Trust, Maidstone Hospital Postgrad Centre, Hermitage Lane, Maidstone, Kent ME169QQ, UK, E-mail: andrew.swindlehurst@doctors.org.uk

Received October 16, 2011; Accepted March 24, 2012; Published March 29 2012

Citation: Swindlehurst A, Swindlehurst N, Kaul S, Corrigan C (2011) Allergy Services at a Tertiary Referral Centre: A One Year Retrospective Review of All Referrals“A One Year Review of Allergy Referrals”. J Aller Ther 3:115. doi:10.4172/2155-6121.1000115

Copyright: ( 2011 Swindlehurst A, et al. This is an open-access article distributed under the terms of the Creative Commons Attribution License, which permits unrestricted use, distribution, and reproduction in any medium, provided the original author and source are credited. 
diagnoses, the diagnosis responsible for the presenting symptom was listed (for example if a patient presenting with food allergy was found to be allergic to egg but was also found incidentally to have grass pollen-induced allergic rhinitis, egg allergy was listed the diagnosis).

7. Management plan (from the clinic letter written by the allergist to the referring doctor). Positive interventions included instances where the allergist prescribed, advised or actively continued an existing treatment and/or provided or referred for specialist allergy investigations, allergen avoidance or immunotherapy.

8. Referral to other specialists (from the clinic letter written by the allergist to the referring doctor).

\section{A priori classification of symptoms}

Symptom categories recorded were determined in advance as follows:

(a) Multi-systemic involvement: combinations of non-lifethreatening symptoms spanning more than one end organ (examples included headache with nausea and flu like symptoms; nasal congestion and blotchy skin; diarrhoea and vomiting with paraesthesia of the extremities; skin and mouth blistering with abdominal cramps).

(b) Skin: dermatitis, pruritus, hives, urticaria, angioedema.

(c) Ear nose \& throat (ENT): rhinitis, rhinorrhoea, nasal itching, sneezing, nasal blockage.

(d) Life-threatening (LT): collapse, loss of consciousness, laryngeal oedema, and severe bronchospasm. When recording LT incidents we required that the history or other documented evidence confirmed or strongly suggested these features: A\&E attendance or administration of adrenaline did not automatically qualify.

(e) Oral: any symptoms involving the lips, tongue and oral cavity.

(f) Gastrointestinal: diarrhoea, nausea, vomiting, abdominal pain, flatus.

(g) Chest/cardiac: palpitations, dyspnoea, shortness of breath, chest tightness, cough, wheezing.

(h) Eye: itching, induration and lachrymation.

(i) Neurological: headache.

\section{Data analysis}

Data were collected directly from computerised hospital records and converted immediately to an anonymised numerical/categorical format and analysed on a Microsoft Excel ${ }^{\oplus}$ spreadsheet. Data collected included:

1. Numbers, age and sex of the patients.

2. Reason(s) for referral in priority order.

3. The working diagnosis/diagnoses at referral prior to any investigations.

4. Investigations requested and how many were abnormal.

5. The final diagnosis/diagnoses by the allergist.

6. Active management by the allergist (commencing or recommending new drugs, referral to further allergy specialist service including dietician).
7. Final disposition of the patients (discharged or referred to other services)

Because the data were gathered anonymously directly from hospital records with strict preservation of patient confidentiality, ethical approval for the study was not deemed necessary.

\section{Results}

The total numbers of patients referred to the allergy service between $1^{\text {st }}$ January and $31^{\text {st }}$ December 2006 grouped by age range and sex are shown in Table 1 (it should be noted that these data are not reflective of paediatric referral activity: patients under 16 year of age should have been referred to the trust paediatric allergy service. This study includes referrals made to the adult allergy clinic only). $73 \%$ of the patients were female. Patients between 21 and 40 years of age comprised 53\% of the total.

Table 2 documents, according to the sources of referral, the percentages of patients suspected to have a possible allergic disease at presentation and those in which this was subsequently proven following all testing (which might have been completed on the day or required one or more extra visits and/or challenge). Diagnostic concordance was relatively good with referrals from the departments of ENT, anaesthetics and immunology. Referrals from dermatology,

\begin{tabular}{|c|c|c|c|c|}
\hline & & $\begin{array}{c}\text { Female } \\
N(\%)\end{array}$ & $\begin{array}{c}\text { Male } \\
N(\%)\end{array}$ & Total \\
\hline Number of patients & & $1245(73.2)$ & $457(26.9)$ & 1702 \\
\hline & & & & \\
\hline Age Category & $0-10$ & 12 & 9 & 21 \\
\hline & $11-20$ & 105 & 30 & 135 \\
\hline & $21-30$ & 365 & 112 & 477 \\
\hline & $31-40$ & 298 & 119 & 417 \\
\hline & $41-50$ & 239 & 78 & 317 \\
\hline & $51-60$ & 122 & 57 & 179 \\
\hline & $61-70$ & 61 & 40 & 101 \\
\hline & $71-80$ & 34 & 10 & 44 \\
\hline & $81+$ & 9 & 2 & 11 \\
\hline Age $(M \pm S D)$ & & $37.8+14.8$ & $39.8+15.8$ & $38.4+17.1$ \\
\hline
\end{tabular}

$N=$ number of patients, $M=$ mean, $S D=$ standard deviation

Table 1: Demographic data of all new patients referred to the allergy service in 2006.

\begin{tabular}{|c|c|c|c|c|}
\hline $\begin{array}{c}\text { Source of } \\
\text { referral }\end{array}$ & $\begin{array}{c}\text { Number of } \\
\text { referrals }\end{array}$ & $\begin{array}{c}\text { Allergist agreed } \\
\text { possible allergy } \\
\text { diagnosis }(\%)\end{array}$ & $\begin{array}{c}\text { Allergist confirmed } \\
\text { allergy diagnosis } \\
(\%)\end{array}$ \\
\hline GP & All & $\begin{array}{c}1426(84 \%) \\
(16 \%)\end{array}$ & 52.2 & 35.1 \\
\hline Hospital & Dermatology & 73 & 58.9 & 37.0 \\
\hline & ENT & 64 & 85.9 & 48.0 \\
\hline & A \& E & 25 & 40.0 & 20.0 \\
\hline & Anaesthetics & 23 & 78.3 & 56.5 \\
\hline & $\begin{array}{c}\text { Gastroenterol- } \\
\text { ogy }\end{array}$ & 13 & 53.9 & 30.8 \\
\hline & Immunology & 7 & 85.7 & 42.6 \\
\hline & Others & 71 & 50.7 & 28.2 \\
\hline
\end{tabular}

${ }^{1}$ Dentistry, respiratory, lupus unit, obstetrics and gynaecology, dietetics, rheumatology, occupational health, maxillofacial surgery, haematology, cardiology, paediatrics, sexual health, ophthalmology, urology, pharmacology, orthopaedics, psychiatry and an allergy department from another site.

Table 2: Allergy diagnosis and management according to the source of referral. 
$\mathrm{A} \& \mathrm{E}$, gastroenterology, other hospital departments and primary care had a poorer diagnostic concordance.

The range of presenting symptoms of referred patients is shown in

\begin{tabular}{|c|c|}
\hline Presenting Complaint $^{*}$ & Number of Patients (\%) \\
\hline Multi-system involvement & $756(44.4)$ \\
\hline Skin & $420(24.7)$ \\
\hline ENT & $188(11.1)$ \\
\hline Life -threatening & $108(6.4)$ \\
\hline Oral & $100(5.9)$ \\
\hline Gastrointestinal & $83(4.9)$ \\
\hline Chest/ cardiac & $27(1.6)$ \\
\hline Eye & $19(1.1)$ \\
\hline Neurology & $1(0.06)$ \\
\hline
\end{tabular}

*Multi-system involvement: combinations of non-life-threatening symptoms spanning more than one end organ; Skin: dermatitis, pruritus, hives, urticaria, angioedema; ENT (ear, nose \& throat): rhinitis, rhinorrhoea, nasal itching, sneezing, nasal blockage; Life-threatening: collapse, loss of consciousness, laryngeal oedema severe bronchospasm; Oral: any symptoms involving the lips, tongue and oral cavity; Gastrointestinal: diarrhoea, nausea, vomiting, abdominal pain, flatus; Chest/ cardiac: palpitations, dyspnoea, chest tightness, cough, wheezing; Eye: itching, induration and lachrymation; Neurological: headache.

Table 3: Presenting complaints of new patients referred to the allergy clinic.

\begin{tabular}{|c|c|c|c|c|c|}
\hline Working diagnoses & $\begin{array}{c}\text { Female } \\
\mathrm{N}\end{array}$ & $\begin{array}{c}\text { Male } \\
\mathrm{N}\end{array}$ & $\begin{array}{c}\text { Total } \\
\mathrm{N}\end{array}$ & $\begin{array}{c}\text { Working } \\
\text { diagnosis } \\
\text { confirmed (\%) }\end{array}$ & $\begin{array}{c}\text { Other } \\
\text { allergy } \\
\text { diagnosec } \\
(\%)^{1}\end{array}$ \\
\hline \multicolumn{6}{|l|}{ Allergic } \\
\hline Food allergy & 269 & 88 & 357 & 47.1 & 7.0 \\
\hline Perennial allergic rhinitis & 132 & 57 & 189 & 58.7 & 7.4 \\
\hline Seasonal allergic rhinitis & 46 & 46 & 92 & 89.1 & 3.3 \\
\hline Other drug allergy ${ }^{2}$ & 57 & 20 & 77 & 50.7 & 3.0 \\
\hline Oral allergy syndrome & 44 & 10 & 54 & 94.4 & 0 \\
\hline Antibiotic allergy & 41 & 8 & 49 & 18.4 & 2.0 \\
\hline Insect venom allergy & 22 & 12 & 34 & 79.4 & 5.9 \\
\hline Latex allergy & 20 & 0 & 20 & 45.0 & 0 \\
\hline Animal dander allergy & 14 & 6 & 20 & 65.0 & 5.0 \\
\hline $\begin{array}{l}\text { Allergen induced anaphy- } \\
\qquad \text { laxis }^{3}\end{array}$ & 7 & 3 & 10 & 80.0 & 0 \\
\hline Allergic urticaria & 8 & 1 & 9 & 44.4 & 0 \\
\hline Nickel allergy & 5 & 0 & 5 & 40.0 & 0 \\
\hline Semen allergy & 2 & 0 & 2 & 50.0 & 0 \\
\hline Contrast media allergy ${ }^{4}$ & 1 & 0 & 1 & 0.0 & 0 \\
\hline Allergic conjunctivitis & 1 & 0 & 1 & 100.0 & 0 \\
\hline $\begin{array}{l}\text { All allergic working diag- } \\
\text { noses }\end{array}$ & & & $\begin{array}{c}920 \\
(54.0 \%)\end{array}$ & & \\
\hline \multicolumn{6}{|l|}{ Non-allergic } \\
\hline Miscellaneous ${ }^{5}$ & 293 & 87 & 380 & 98.4 & 1.6 \\
\hline Idiopathic urticaria & 189 & 77 & 266 & 95.1 & 2.3 \\
\hline Idiopathic angioedema & 78 & 30 & 108 & 92.6 & 3.7 \\
\hline Idiopathic anaphylaxis & 8 & 7 & 15 & 66.7 & 0 \\
\hline Cholinergic urticaria & 7 & 4 & 11 & 100.0 & 0 \\
\hline Dermographic urticaria & 1 & 1 & 2 & 100.0 & 0 \\
\hline $\begin{array}{c}\text { All non-allergic working } \\
\text { diagnoses }\end{array}$ & & & $\begin{array}{c}782 \\
(46.0 \%)\end{array}$ & & \\
\hline
\end{tabular}

${ }^{1}$ Working diagnosis not confirmed but other allergic disease diagnosed

${ }^{2}$ Anaesthetics, muscle relaxants, NSAIDs, aspirin, paracetamol, morphine, chlorhexidine, gabapentin, gelofusin

${ }^{3}$ Does not appear in Table 5: listed according to causative allergen or classified as non-allergic

${ }^{4}$ Does not appear in table 5: not verified

Includes symptoms attributed to food "intolerance", non-atopic eczema, asthma and other non-allergic conditions

Table 4: Working diagnoses made by the allergist after the initial appointment.
Table 3. Most patients complained of non-life threatening symptoms involving multiple organ systems. Complaints confined to one organ system involved the skin most commonly, followed by ENT, oral and gastrointestinal. Isolated chest/cardiac, ocular and neurological symptoms were uncommon. $6.4 \%$ of referrals were for what allergists perceived as potentially life threatening symptoms.

Table 4 lists all of the working diagnoses made by allergists based on the impressions of the referring physicians or other health professionals, other information in the referral letters and investigations the results of which were available at the initial clinic visit (typically only skin prick tests). A working diagnosis of allergic disease was made in 920 (54\%) of the referred patients; the remaining 782 (46\%) patients were thought to have non-allergic diagnoses. About half of the non-allergic diagnoses were idiopathic urticaria and/or angioedema, and the remainder were a miscellaneous assortment of conditions but in particular non-allergic rhinosinusitis or asthma, non-specific gastrointestinal and other symptoms attributed to food "intolerance" and skin rashes other than eczema or urticaria.

Table 5 lists all final diagnoses and the percentages of patients in each working diagnostic category who had their final diagnoses changed following complete investigation. Only 592 (34.8\%) of the final diagnoses were of allergic disease. Significant proportions of patients (44.2-75.5\%) provisionally diagnosed with food, drug and latex allergy and rarer allergies such as semen allergy had an allergic aetiology ruled out following investigation. Similarly, more cases of "idiopathic" urticaria, angioedema and anaphylaxis, asthma and eczema and many more cases of non-specific gastrointestinal symptoms and chronic rhinosinusitis were determined as having a non-allergic aetiology.

Outcomes following the patients' first appointments are summarised in Table 6 . Overall, $54.2 \%$ of patients were discharged following their first clinic visit. Interestingly, approximately equal percentages of patients who did and did not eventually prove to have allergic disease were followed up in the general allergy clinic $(25.2 \%$ vs $26.0 \%$ ) or referred for specialist allergy investigations (drug challenge $4.2 \%$ vs $5.6 \%$; allergy specialist dietetic referral $4.9 \%$ vs $3.6 \%$; food challenge $0.8 \%$ vs $1.5 \%$ respectively). $12.2 \%$ of patients diagnosed with allergic disease were referred for immunotherapy.

A list of all investigations performed and the proportions which were abnormal is shown in Table 7. A total of 2,354 sets of blood investigations were ordered. Overall $21.6 \%$ of these showed some abnormality, although not necessarily of pathological significance. In vitro allergen-specific IgE tests (involving unrecorded numbers of allergens) were ordered on 222 occasions. Skin prick tests were ordered 1,099 times; at least one positive test was observed in $58.6 \%$ of cases. The right hand column in Table 7 shows the numbers of patients (and percentages of the total of 922) discharged after their first clinic visit having had each test. Overall 184 of these patients $(20.0 \%$ of the total) had at least one blood test. Drug challenge was performed on 87 patients and was positive in 15 (17.2\%); 22 patients were challenged with foods, 2 (9.1\%) of whom had a positive reaction.

\section{Discussion}

We present a retrospective analysis of all 1,702 new patient referrals to a tertiary allergy referral centre of the course of one entire year. $84 \%$ of referrals were from primary care; $16 \%$ were from hospital departments other than allergy, while $<1 \%$ were from allergy departments in other hospitals. Females were referred 3 times more frequently than males, although the age distributions of referred females and males 
Citation: Swindlehurst A, Swindlehurst N, Kaul S, Corrigan C (2011) Allergy Services at a Tertiary Referral Centre: A One Year Retrospective Review of All Referrals“A One Year Review of Allergy Referrals". J Aller Ther 3:115. doi:10.4172/2155-6121.1000115

Page 4 of 6

\begin{tabular}{|c|c|c|c|c|c|c|}
\hline Final diagnoses & $\underset{\mathrm{N}}{\text { Female }}$ & $\stackrel{\text { Male }}{N}$ & $\begin{array}{c}\text { Total } \\
\mathrm{N}\end{array}$ & $\begin{array}{l}\text { Concordance between working } \\
\text { and final diagnosis }(\%)^{1}\end{array}$ & $\begin{array}{l}\text { New drugs prescribed } \\
\text { or advice given (\%) }\end{array}$ & $\begin{array}{l}\text { New management (other } \\
\text { than drugs) }(\%)^{2}\end{array}$ \\
\hline \multicolumn{7}{|l|}{ Allergic } \\
\hline Food allergy & 121 & 57 & 178 & -50.1 & 60.7 & 12.4 \\
\hline Perennial allergic rhinitis & 78 & 38 & 116 & -38.6 & 65.5 & 23.3 \\
\hline Seasonal allergic rhinitis & 51 & 46 & 97 & 5.4 & 61.9 & 37.1 \\
\hline Oral allergy syndrome & 65 & 13 & 78 & 44.4 & 53.9 & 10.3 \\
\hline Insect venom allergy & 19 & 13 & 32 & -5.9 & 59.4 & 56.3 \\
\hline Animal dander allergy & 11 & 4 & 15 & -25.0 & 80.0 & 26.7 \\
\hline Antibiotic allergy & 10 & 2 & 12 & -75.5 & 0 & 75.0 \\
\hline Other drug allergy ${ }^{3}$ & 31 & 12 & 43 & -44.2 & 16.3 & 30.2 \\
\hline Latex allergy & 11 & 0 & 11 & -45.0 & 45.5 & 18.2 \\
\hline Allergic urticaria & 4 & 1 & 5 & -44.4 & 80.0 & 40.0 \\
\hline Nickel allergy & 2 & 0 & 2 & -60.0 & 0 & 100.0 \\
\hline Delayed allergic reaction ${ }^{4}$ & 1 & 0 & 1 & - & 100.0 & 0 \\
\hline Semen allergy & 1 & 0 & 1 & -50.0 & 0 & 100.0 \\
\hline Allergic conjunctivitis & 1 & 0 & 1 & 0.0 & 100.0 & \\
\hline All allergic final diagnoses & 406 & 186 & $592(34.8)$ & & & \\
\hline \multicolumn{7}{|l|}{ Non-allergic } \\
\hline Miscellaneous ${ }^{5}$ & 523 & 144 & 667 & 75.5 & 23.5 & 22.8 \\
\hline Idiopathic urticaria & 205 & 79 & 284 & 6.8 & 58.8 & 9.9 \\
\hline Idiopathic angioedema & 90 & 32 & 122 & 13.0 & 45.5 & 11.5 \\
\hline Idiopathic anaphylaxis & 10 & 8 & 18 & 20.0 & 66.7 & 16.7 \\
\hline Cholinergic urticaria & 9 & 7 & 16 & 45.5 & 68.8 & 6.3 \\
\hline Dermographic urticaria & 2 & 1 & 3 & 50.0 & 66.7 & 0 \\
\hline All non-allergic final diagnoses & 839 & 271 & $1110(65.2)$ & & & \\
\hline
\end{tabular}

${ }^{1}$ Percentages of patients given this working diagnosis in Table IV who were given a different final diagnosis following full investigation. A negative value indicates that there were more patients in the working diagnosis than final diagnosis category

${ }^{2}$ Drug challenge testing, food challenge testing, allergen immunotherapy, desensitisation, allergen avoidance including expert dietetic advice, referral to another speciality ${ }^{3}$ Anaesthetics, muscle relaxants, NSAIDs, aspirin, paracetamol, morphine, chlorhexidine, gabapentin, gelofusin

${ }^{4}$ Classified as insect venom allergy in Table IV but eventually diagnosed as "delayed allergic reaction"

5Includes symptoms attributed to food "intolerance", non-atopic eczema, asthma and other non-allergic conditions. Table 5: Final diagnoses of new patients referred to the allergy clinic.

\begin{tabular}{|c|c|c|c|}
\hline & $\begin{array}{l}\text { Allergy } \\
\mathrm{N}(\%)^{1}\end{array}$ & $\begin{array}{c}\text { Non-allergy } \\
\mathrm{N}(\%)^{1}\end{array}$ & $\begin{array}{l}\text { Total } \\
\mathrm{N}(\%)^{2}\end{array}$ \\
\hline \multicolumn{4}{|l|}{ Outcome of first appointment: } \\
\hline (1) Discharged & $300(50.1 \%)$ & $622(56.1 \%)$ & $922(54.2 \%)$ \\
\hline (2) Followed up in allergy clinic & $149(25.2 \%)$ & $289(26.0 \%)$ & $438(25.7 \%)$ \\
\hline (3) Referred to another speciality & $15(2.5 \%)$ & $79(7.1 \%)$ & $94(5.5 \%)$ \\
\hline (4) Referred for drug challenge & $25(4.2 \%)$ & $62(5.6 \%)$ & $87(5.1 \%)$ \\
\hline (5) Referred for immunotherapy & $72(12.2 \%)$ & 0 & $72(4.2 \%)$ \\
\hline (6) Referred to dietician & $26(4.9 \%)$ & $40(3.6 \%)$ & $67(3.9 \%)$ \\
\hline (7) Referred for food challenge & $5(0.8 \%)$ & $17(1.5 \%)$ & $22(1.3 \%)$ \\
\hline \multicolumn{4}{|l|}{ Referred at any time during management: } \\
\hline (1) Referred to dietician & $30(5.1 \%)$ & $59(5.3 \%)$ & $89(5.2)$ \\
\hline (2) Referred for immunotherapy & $78(13.2 \%)$ & 0 & $78(4.6)$ \\
\hline \multicolumn{4}{|l|}{ Medication: } \\
\hline Prescribed, recommended or advice given & $335(56.6 \%)$ & $430(38.7 \%)$ & $765(44.9 \%)$ \\
\hline None prescribed or recommended & $257(43.4 \%)$ & $680(61.3 \%)$ & $937(55.1 \%)$ \\
\hline
\end{tabular}

${ }^{1}$ Percentages are of the total patients with allergic $(592)$ and non-allergic $(1,110)$ final diagnoses (TableV)

${ }^{2}$ Percentages of all 1,702 patients

Table 6: Further management of all new patients following their first appointments.

were very similar, with a mean referral age of 38 years (Table 1 ). The frequency with which allergists agreed with the provisional referral diagnosis of allergy varied with the source of referral: concordance was good with referrals from ENT (84.9\%), anaesthetics (78.3\%) and immunology $(85.7 \%)$, but there was less concordance with primary care referrals (52.2\%) and referrals from dermatology (58.9\%), A\&E (40.0\%), gastroenterology (53.9\%) and other hospital departments
(50.7\%). The percentage of diagnostic concordance will obviously reflect the range of possible allergy diagnoses encountered by the referring physicians as well as their allergy experience. It is of concern however that diagnostic concordance was lower with primary care and A\&E physicians, since these stands in the "front line" of allergy management in the community. This likely reflects the fact that allergy is not a fundamental topic included in the undergraduate or junior 


\begin{tabular}{|c|c|c|c|}
\hline Investigation & Total performed & Total abnormal $(\%)^{1}$ & Discharged $(\%)^{2}$ \\
\hline Full blood count & 312 & $116(37.2)$ & $124(13.4)$ \\
\hline Auto-antibodies $^{3}$ & 269 & $21(7.8)$ & $109(11.8)$ \\
\hline Erythrocyte sedimentation rate & 261 & $50(19.2)$ & $106(11.5)$ \\
\hline Allergen-specific $\lg E$ & 222 & $84(37.8)$ & $45(4.9)$ \\
\hline Thyroid function test & 185 & $11(6.0)$ & $72(7.8)$ \\
\hline Complement (C3, C4) & 165 & $12(7.3)$ & $63(6.8)$ \\
\hline Liver profile & 164 & $29(17.7)$ & $57(6.2)$ \\
\hline $\mathrm{C}$ reactive protein & 152 & $34(22.4)$ & $64(6.9)$ \\
\hline Renal Profile & 152 & $26(17.1)$ & $58(6.3)$ \\
\hline Serum $\lg \mathrm{E}$ & 112 & $68(60.7)$ & $35(3.8)$ \\
\hline Bone profile & 98 & $13(13.3)$ & $38(4.1)$ \\
\hline Immunoglobulins & 63 & $24(38.1)$ & $35(3.8)$ \\
\hline Serum electrophoresis & 60 & $1(1.7)$ & $29(3.1)$ \\
\hline C1 esterase inhibitor & 55 & $0(0)$ & $34(3.7)$ \\
\hline Rheumatoid factor & 42 & $10(23.8)$ & $4(0.4)$ \\
\hline Lipid profile & 20 & $6(30)$ & $8(0.9)$ \\
\hline Glucose & 11 & $0(0)$ & $3(0.3)$ \\
\hline H. Pylori antibodies & 7 & $3(42.9)$ & $2(0.2)$ \\
\hline Clotting screen & 4 & $0(0)$ & $3(0.3)$ \\
\hline Skin prick tests ${ }^{4}$ & 1099 & $644(58.6)$ & $596(64.6)$ \\
\hline Chest X-ray & 18 & $3(16.7)$ & $5(0.5)$ \\
\hline Drug challenge & 87 & $15(17.2)$ & - \\
\hline Food challenge & 22 & $2(9.1)$ & - \\
\hline CT scan sinuses & 16 & $11(68.8)$ & - \\
\hline Lung function testing & 13 & $4(30.8)$ & $2(0.2)$ \\
\hline
\end{tabular}

${ }^{1}$ Any positive categorical result or any measurement outside the reference range

${ }^{2}$ Numbers of patients and percentages of the total 922 patients discharged after the first clinic appointment having had this test

Includes thyroid antibodies, ANA and ANCA

${ }^{4}$ Variable total numbers of allergens

Table 7: Range and timing of investigations performed and proportions of abnormal results.

postgraduate medical curricula. Further, the term "concordance" here is perhaps somewhat stark: it is also possible that some patients were referred primarily to confirm the referrer's impression of a non-allergic diagnosis. "Concordance" with all referral sources was even lower after full investigation and final diagnosis (Table 2).

What do allergists do? This study shows that they see people of all ages referred from primary care and a wide variety of hospital specialist departments, sometimes with symptoms confined to a specific organ but more commonly with mixtures of symptoms involving multiple organ systems, particularly the skin, upper and lower respiratory tracts and the upper and lower gastrointestinal tracts. Thus, allergists must be astute not only in identifying allergic causes of symptoms in these organs but also in identifying non-allergic symptoms, particularly those which may be due to malignant disease, or other life-limiting illness, requiring prompt investigation. Allergists should be trained with this in mind. About half of all allergists' referrals are for diseases that are not known to have an allergic aetiology (particularly non-specific bowel symptoms and "idiopathic" urticaria and angioedema), although it is occasionally of vital importance (for example in "idiopathic" anaphylaxis) to rule out an allergic trigger for these diseases. The other half of the referrals are for suspected food allergic reactions, seasonal and perennial allergic rhinoconjunctivitis, oral allergy syndromes, suspected drug allergy, venom allergy, latex allergy and a smattering of other specific diagnoses such as semen allergy the management of which is entirely and exclusively within the realm of the allergist. There are few other studies with which to compare our data. England and colleagues [7] reported inpatient referrals for allergy/immunology over a 14 year period in a tertiary care setting and also reported a significant number of nonallergic diagnoses, but their data are not directly comparable because these were inpatient referrals in the context of a fully developed and widely available allergy service. Jorgensen and Pedersen [8] reported that general practitioners with an interest in allergy were as good as allergists at performing skin prick tests but there was less concordance about final diagnosis, patients suitable for desensitisation and those felt to need more specialist investigations.

What use are allergists? A glance at Tables 6 and 7 shows that about half $(54.2 \%)$ of the annual new referrals were discharged after a single clinic visit during which $64.6 \%$ had skin prick tests and $20 \%$ had at least one blood test but only $0.7 \%$ had any other test (lung function or chest X-ray). Approximately equal proportions of these patients had allergic and non-allergic final diagnoses (Table 6). These patients could theoretically be managed in the community in the future if interested primary care specialists are endowed with the appropriate expertise and have access to skin prick testing, or if specialists bring expertise and skin prick testing with them to outreach clinics. The remainder of the patients were deemed to require further specialist allergy input, either through further follow up (25.7\%), drug challenge (5.1\%), food challenge (1.3\%), allergen avoidance advice including specialist allergy dietetic advice (5.2\%) or allergen immunotherapy (4.6\%). These investigations or services form the "core business" of allergy specialists and should form the backbone of allergy training programmes. A further $5.5 \%$ were deemed to require further management by other hospital specialists (typically for skin, gastrointestinal or respiratory tract symptoms). It is notable that, across the entire spectrum of specialist allergy services shown in Table 6 (with the exception of immunotherapy), approximately equal proportions of patients who had allergy and non-allergy final diagnoses were referred, suggesting that specialist allergy investigation is required to rule out allergy as 
often as it is to confirm it. In addition to specialist services, Tables 5 and 6 also show that allergists provided a majority of patients in nearly all of the final diagnostic categories, allergic or non-allergic, with advice about their medications. This included advice about how to use antihistamines and nasal steroids for hay fever, how to use nasal sprays correctly, provision of anaphylaxis plans including instruction on when and how to use Epipens and the provision of topical adrenaline sprays and management plans for chronic urticaria and angioedema and advice about alternative medications in patients with drug allergy or drug-induced angioedema. Informed allergen avoidance advice, including dietetic advice is also largely provided through allergy services. What use are analyses like this one? It has been emphasised that two obvious outcomes are to guide training of allergists and to mould services, both hospital and community based to meet the needs of patients. Keeping a watching brief on what allergists are required to do may also inform revalidation. Ultimately, strategically placed studies at major centres will allow estimation of the size of the allergy problem. It is noteworthy that the Department of Health review of allergy services [2] made the point that "the absence of baseline data on the profile of allergy services and the cost makes it difficult to develop a strategic national view of how and where services could be developed". Studies such as this one generate baseline data and hypotheses for further interventional studies, assessing impact on patient experience and altering the focus of the service to meet the needs of the end-user.

The diagnostic value of the investigative processes shown in table 7 is difficult to assess from a simple list but such lists are useful pointers towards more focussed audit as to the appropriateness of "routine" investigations such as full blood counts and in vitro IgE testing compared with skin prick testing.

The data in this study are already ageing: in the 4 years since 2006 our referrals for drug and food allergy and immunotherapy have continued to rise and we now provide an inpatient drug allergy diagnostic and advice service. We were obliged to go back several years so as to be able to analyse and track the final outcomes of all of the patients. This increased the robustness of the findings but delayed their retrieval. We did not undertake formal reproducibility studies but feel that the high concordance of the data collected simultaneously but independently by two investigators using agreed criteria further adds to the robustness of the data. Ideally such studies should be ongoing.

\section{References}

1. Gupta R, Sheikh A, Strachan DP, Anderson HR (2004) Burden of allergic disease in the UK: secondary analyses of national databases. Clin Exp Allergy 34: $520-526$.

2. Department of Health (2006)A Review of services for allergy: The epidemiology demand for and provision of treatment and effectiveness of clinical interventions.

3. Royal College of Physicians (2003) Allergy: the unmet need A blueprint for better patient care.

4. http://www.publications.parliament.uk/pa/cm200304/cmselect/ cmhealth/696/69602.htm

5. Lord Darzi (2008) High Quality Care for all.

6. Ewan PW (2000) Provision of allergy care for optimal outcome in the UK. Br Med Bull 56: 1087-1101.

7. England RW, Ho TC, Napoli DC, Quinn JM (2003) Inpatient consultation of allergy/immunology in a tertiary care setting. An Allergy Asthma Immunol 90: 393-397.

8. Jørgensen AF, Pedersen PA (1999) Allergological examination in genera practice and in the specialist field of allergology. A comparative study of the concordance between a group of general practitioners and the allergological specialised health care services to which they refer. Scand J Prim Health Care 17: 221-225. 